\title{
Host and Symbiont Jointly Control Gut Microbiota during Complete Metamorphosis
}

\author{
Paul R. Johnston ${ }^{1 *}$, Jens Rolff ${ }^{1,2 *}$ \\ 1 Freie Universität Berlin, Berlin, Germany, 2 Berlin-Brandenburg Institute of Advanced Biodiversity \\ Research (BBIB), Berlin, Germany \\ * paul.johnston@fu-berlin.de (PRJ); jens.rolff@fu-berlin.de (JR)
}

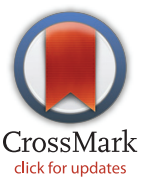

\section{G openaccess}

Citation: Johnston PR, Rolff J (2015) Host and Symbiont Jointly Control Gut Microbiota during Complete Metamorphosis. PLoS Pathog 11(11): e1005246. doi:10.1371/journal.ppat.1005246

Editor: David S. Schneider, Stanford University, UNITED STATES

Received: June 1, 2015

Accepted: October 2, 2015

Published: November 6, 2015

Copyright: @ 2015 Johnston, Rolff. This is an open access article distributed under the terms of the Creative Commons Attribution License, which permits unrestricted use, distribution, and reproduction in any medium, provided the original author and source are credited.

Data Availability Statement: The data are available at NCBI SRA under accession PRJNA268795.

Funding: This research was supported by European Research Council (http://erc.europa.eu/) grant 260986 to J.R. and by the BBSRC Genome Analysis Centre Capacity \& Capability Challenge program to JR and PRJ (http://www.bbsrc.ac.uk/). The funders had no role in study design, data collection and analysis, decision to publish, or preparation of the manuscript.

Competing Interests: The authors have declared that no competing interests exist.

\section{Abstract}

Holometabolous insects undergo a radical anatomical re-organisation during metamorphosis. This poses a developmental challenge: the host must replace the larval gut but at the same time retain symbiotic gut microbes and avoid infection by opportunistic pathogens. By manipulating host immunity and bacterial competitive ability, we study how the host Galleria mellonella and the symbiotic bacterium Enterococcus mundtii interact to manage the composition of the microbiota during metamorphosis. Disenabling one or both symbiotic partners alters the composition of the gut microbiota, which incurs fitness costs: adult hosts with a gut microbiota dominated by pathogens such as Serratia and Staphylococcus die early. Our results reveal an interaction that guarantees the safe passage of the symbiont through metamorphosis and benefits the resulting adult host. Host-symbiont "conspiracies" as described here are almost certainly widespread in holometobolous insects including many disease vectors.

\section{Author Summary}

The majority of animals are holometabolous insects and change dramatically through development. They undergo a dramatic transformation from a larval stage, adapted to feed, to an adult separated by a pupal stage. During this pupal stage the majority of the organs are renewed including the gut. This creates a risky situation that we study here: when the gut is renewed insects risk losing beneficial microbiota while simultaneously being at risk of opportunistic infection. Here, by manipulating host and symbiont we show how host and symbiont succeed in jointly controlling opportunistic pathogens. If one or both of the partners are compromised, opportunistic pathogens dominate the gut microbiota resulting in increased mortality. These findings may be broadly applicable to insects with complete metamorphosis, including many disease vectors.

\section{Introduction}

The vast majority of animal species are insects [1,2]. Most species of insects are holometabolous, including important vectors of infectious disesases (sandflies, mosquitoes) and model 
organisms (Drosophila melanogaster, Galleria mellonella) with distinct larval and adult stages separated by metamorphosis, which entails dramatic remodeling of external and internal anatomy $[3,4]$. The evolutionary advantage of metamorphosis is usually explained by the adaptive decoupling hypothesis [5]: traits in larvae and adults are genetically decoupled, facilitating adaptation to life-stage specific selection [6]. Anatomical re-organization of the body, however, poses a significant problem during the replacement of the gut, as the gut hosts a microbiota. Either the organism must eradicate and subsequently re-establish the microbiota from the environment, or it must maintain its microbiota while preventing opportunistic microbes from entering the hemocoel and causing infections.

Early studies clearly demonstrated the presence and maintenance of bacteria in the gut during metamorphosis in Lepidoptera and Diptera [7-9], and more recent work has described the same phenomenon in Coleoptera [10], Diptera [11], Lepidoptera [12], and Hymenoptera [13]. Two competing mechanisms have been proposed to explain the composition of the retained gut microbiota. One explanation holds that bacterial competition drives the composition of the adult gut microbiota $[14,15]$. Alternatively, the host immune system plays an important role in shaping the gut microbiota $[16,17]$. However despite continued interest in insect gut immunity [18], the interaction between host immunity and bacterial competition during complete metamorphosis has remained unstudied.

Here we exploit an ancient [19], facultative and prevalent [20-26] symbiosis between Lepidoptera and enterococci to reconcile these approaches by studying the role of host immunity and bacterial competition during metamorphosis within a single system. The gut microbiota of Lepidoptera is limited to a handful of bacterial species that varies with habitat and diet but often is dominated by enterococci that persist through metamorphosis [12]. In the lepidopteran gut, enterococci interact with pathogens through (i) competitive exclusion (ii) attenuation by direct antagonism or (iii) eliciting protective host immune responses and provide lepidopterans including Galleria mellonella with protection against one of the most virulent entomopathogens, Bacillus thuringiensis [21,27-29] (reviewed in [30]).

In Lepidoptera, the contents of the gut lumen and the peritrophic matrix are purged at the onset of metamorphosis. Basal midgut stem cells proliferate and differentiate to form a continuous layer beneath the larval gut epithelium where lysozyme accumulates in apical vacuoles [16]. Following ecdysis of the larval epithelium, the vacuole contents are released into the gut lumen producing a burst of antibacterial activity that is presumed to prevent septicemic infection [16]. The detached larval epithelium undergoes autophagy and apoptosis and degenerates as the 'yellow body' [31]. Bacteria that resist mechanical and immunological exclusion by the host during pupation, compete intensely for colonization of the pupal gut as has been demonstrated in flies [14].

In the model lepidopteran Galleria mellonella, Enterococcus mundtii (syn. Streptococcus faecalis Andrewes and Horder) is passed from female to offspring via the surface of the egg [25]. In vitro observations of lepidopteran gut microbes imply that $E$. mundtii has the highest abundance, in adults it is often the only detectable microbe in the gut, and that this is mediated by synergy between lysozyme and a broad-spectrum bacteriocin [28]. As is common in many Lepidoptera [32], G. mellonella adults lack functional mouthparts and therefore additional microbes cannot be acquired during adult life.

Using the Galleria-Enterococcus symbiosis we tested the hypothesis that host and symbiont interact to determine the adult gut microbiota. We manipulated host gut immunity and bacterial competitive ability during metamorphosis in a full factorial fashion. Based on these findings we studied fitness consequences of altered microbiotas for adult hosts. 


\section{Results and Discussion}

Previous studies show that lysozyme is important in host-microbe interactions in the pre-pupae of another lepidopteran [16], and in G. mellonella a synergistic interaction between C-type lysozyme and antimicrobial peptides was recently demonstrated [33]. Based on the expression of a C-type lysozyme (Swiss-Prot accession P82174) in the gut during metamorphosis (S1 Fig) and the reported synergism of G. mellonella lysozyme we hypothesized that lysozyme mediates changes in the microbiota during metamorphosis. We knocked down lysozyme expression using RNAi in insect hosts that were colonized by either E. mundtii strain G2-mun+, which produces the broad-spectrum bacteriocin mundticin [34], or by E. mundtii strain G2-mun-, which is unable to express the mundticin-encoding gene munA.

To test the impact of these manipulations we cured the gut microbiota from final-instar larvae using antibiotics and re-inoculated these individuals with either E. mundtii G2-mun+ or G2-mun-, and reared them on conventional non-sterile diet until pupation.

Using a combination of $16 \mathrm{~S}$ rRNA gene amplicon sequencing, qPCR, and conventional bacterial culturing we monitored the composition of the gut microbiota during the larval-pupal molt as well as after adult eclosion.

The microbiota of the wild-type host during pupation was increasingly dominated by Enterococcus, whereas Serratia and Staphylococcus were undetectable in the adult stage by culturing, 16S rRNA gene amplicon sequencing (S2 Fig, S1 Table), and 16S rRNA gene qPCR (Fig 1). Host lysozyme-knockdown resulted in a significant increase in Enterobacteriaceae and persistence into the adult stage $(\mathrm{T}=-25.145 \mathrm{df}=456, p=<0.0001)$, which appear to be entirely composed of a Serratia sp. When the host is instead associated with E. mundtii G2-mun- (which does not produce the bacteriocin munditicin), Staphylococcus becomes highly abundant after pupation ( $\mathrm{T}=-96.48, \mathrm{df}=456, p<0.0001$, Fig 1$)$ and Enterococcus are reduced by two orders of magnitude. When both host and symbiont are disenabled, Enterobacteriaceae (Serratia sp.) dominates $(\mathrm{T}=-28.655, \mathrm{df}=456, p<0.0001)$ and again Enterococcus are strongly reduced on reaching the adult stage $(\mathrm{T}=10.290, \mathrm{df}=448, p<0.0001$, Fig 1$)$.

We found that the gut microbiota composition significantly changes when either the symbiont and/or host and symbiont were both disenabled (Fig 1). Based on these results we therefore inoculated mature larvae, that were first cleared of their microbiota using antibiotics ('re-inoculated larvae'), with a defined microbiota comprising either wild-type Enterococcus mundtii, Staphylococcus sp. (reflecting the results when the symbiont is disenabled) or Serratia sp. (as found when both host and symbiont are disenabled), which were isolated from G. mellonella larvae. This made it possible to investigate fitness costs of a defined microbiota without the confounding effects of the manipulation of the host immune system by RNAi or changes to symbiont competitive ability by manipulating mundticin expression. Survival of the resulting adults was monitored after eclosion. Independent of host sex, G. mellonella individuals with $E$. mundtii survived significantly longer than those with a Staphylococcus- $(Z=-4.72, p=<0.0001)$, or Serratia- $(Z=-2.97, p=0.003)$ dominated microbiota (Fig 2, S2 Table). There was no difference in survival between $G$. mellonella adults derived from larvae that were either cured of their microbiota and maintained axenically, or which were re-inoculated with E. mundtii (S3 Fig). This supports the conclusion that the main benefit of E. mundtii mutualism is the interaction with other members of the gut microbiota.

Our study shows that host and symbiont interact to maintain a 'healthy' gut microbiota through complete metamorphosis. Given the protection that E. mundtii confers to the lepidopteran host $[28,30]$ the selective advantage for the host is clear. The transmission of the gut microbiota between individuals is usually considered as mixed-mode transmission, combining vertical and horizontal transmission [35]. For the bacterial symbiont, passage through 


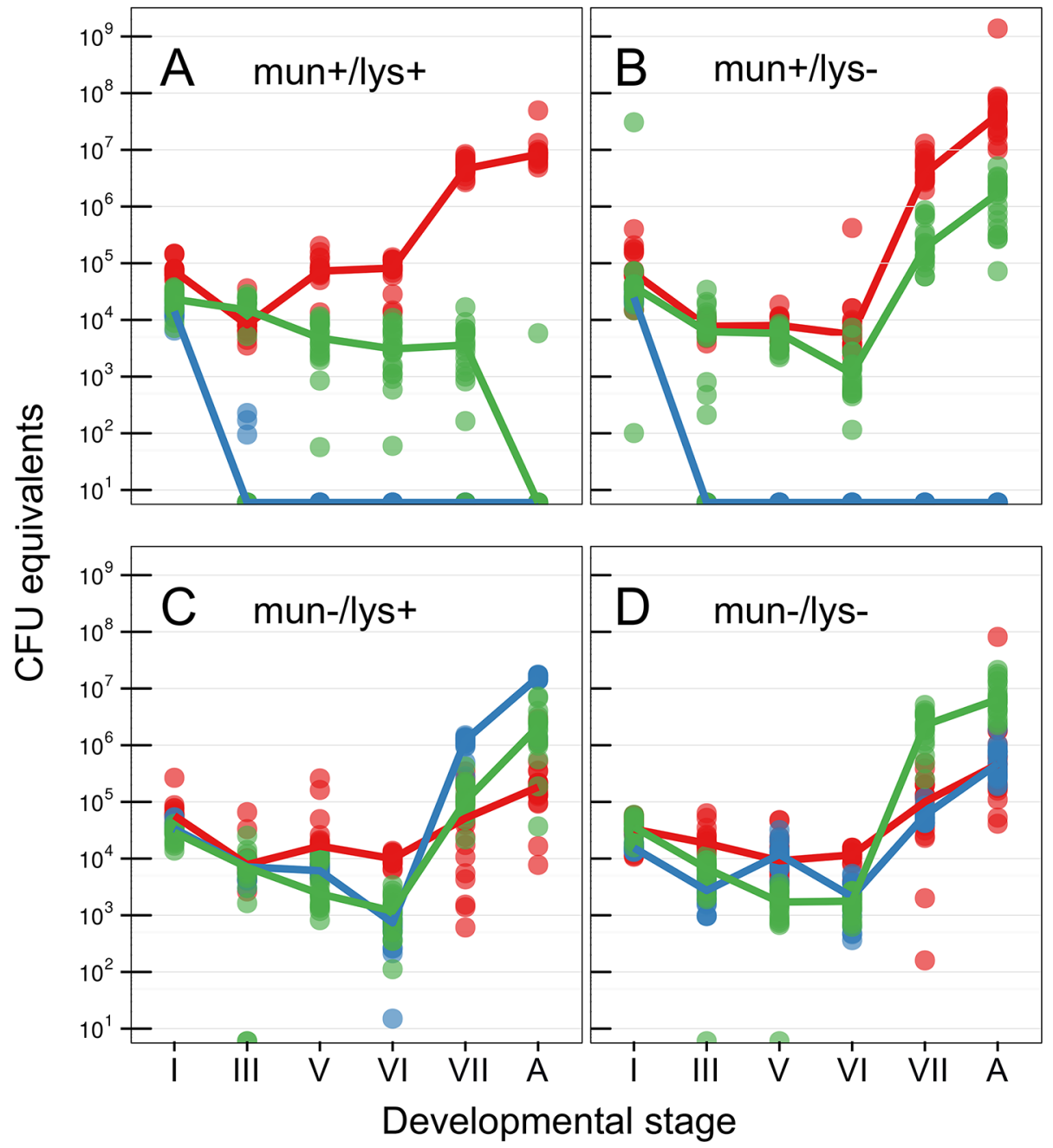

Taxon

- Enterococcus

- Enterobacteriaceae

- Staphylococcus

Fig 1. Abundance of bacteria during host metamorphosis. Using $16 \mathrm{~S}$ rRNA gene qPCR the microbiota was sampled across metamorphosis in (A) wildtype hosts with bacteriocin-producing Entercoccus mundtii G2 symbiont, (B) immunocompromised hosts where RNAi was used to knock down lysozyme gene expression, (C) wild-type hosts with mutant Entercoccus mundtii symbionts that do not produce bacteriocin, and (D) both immunocompromised hosts and mutant symbionts. Roman numerals correspond to precise stages of the larval-pupal molt (see materials and methods). A, adult.

metamorphosis constitutes an important component of vertical transmission. Complete sterilization of the gut by the host would spell disaster for the symbiont. The transition of the symbiont through metamorphosis is crucial to guarantee vertical transmission to the host offspring. In social species, this can be overcome through exposure to feces of nest mates [36]. But most holometabolous insects are solitary and therefore this mechanism of re-acquisition of symbionts from conspecifics after metamorphosis is impractical. Some species of ants and weevils have resolved the transition of the microbiota through metamorphosis with bacteriocytes, crypts, and other specialized structures that harbour symbionts throughout the life cycle $[37,38]$, this is not the case in Lepidoptera.

Our study also sheds light on an important aspect of the evolution of complete metamorphosis, a key innovation of holometabolous insects [39] that resulted in their extraordinary diversity. An understanding of the benefits and costs of complete metamorphosis is essential to explain the evolutionary success of holometabolous insects. Ecological and evolutionary models of metamorphosis are sufficient to explain the evolution of complex life cycles [5], but not 


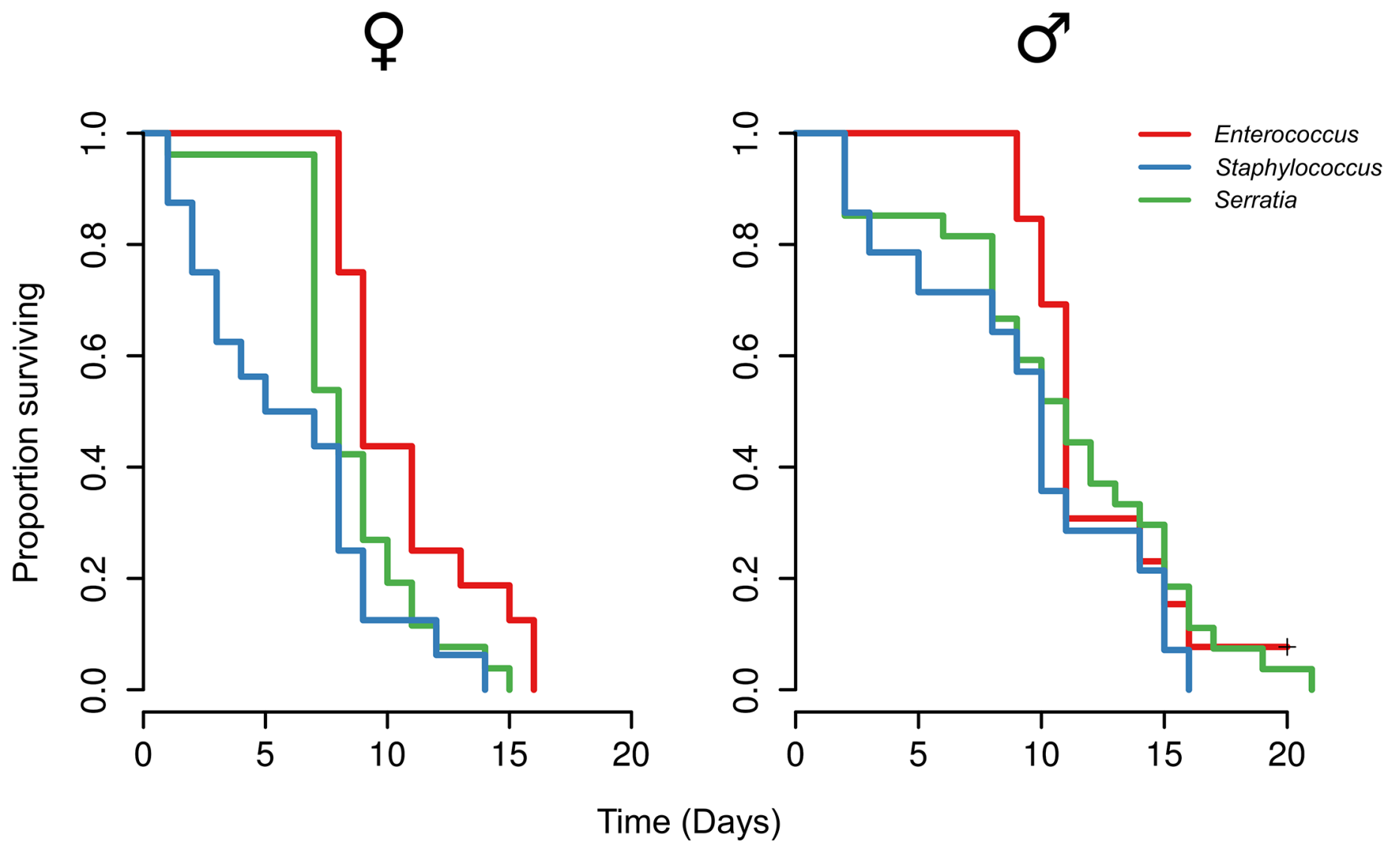

Fig 2. Survival of adults with experimentally defined gut microbiota. Larvae were re-inoculated with a defined microbiota comprising either wild-type Enterococcus mundtii, Staphylococcus sp. or Serratia sp. based on previous results (Fig 1).

doi:10.1371/journal.ppat.1005246.g002

of the pupal stage that defines complete metamorphosis. One adaptive explanation that has been proposed, but barely tested, is the decoupling of growth and differentiation into consecutive stages of the larva and the pupa [40]. The evolution of such a novel complex trait as the pupal stage also creates significant problems. The pupa is a sessile and hence vulnerable stage in the insect life cycle. Some parasitoids specialize on pupal hosts and display adaptations that exploit host endocrinology during metamorphosis [41]. Here, by contrast, we have identified and described a ubiquitous problem encountered by most holometabolous insect during ontogeny: the passage of symbiontic bacteria through pupation. Our data show how the host and symbiont interact to achieve this passage. Moreover, the data also suggest that the risk of opportunistic infection during the destruction of the larval gut is countered by the host through up-regulation of immune function. The destruction of the gut, and hence the abundance of danger signals, in combination with microbe-derived immune elicitors result in a strong immune response [42] as found here.

\section{Materials and Methods}

\section{Bacterial strains and growth conditions}

Enterococcus mundtii G2 was isolated from a long-term laboratory population of Galleria mellonella where insects were reared on a natural diet of honeycomb [43]. To cure E. mundtii G2 of its munditicin-encoding plasmid, a single colony was picked and grown in BHI broth at 
$42^{\circ} \mathrm{C}$ for 524 -h serial passages. The resultant strain was transformed by the method of Dunny et al. [44] with either pRK1 or pRK62 which both contain the entire mun locus with and without a munA promoter, respectively [34]. Both strains express the mundticin ABC transporter protein and mundticin immunity protein (MunB and MunC respectively) [34], and enterococci are intrinsically resistant to lysozyme [45]. The resultant strains are referred to as mun + and mun-. The wild-type mun locus comprises munA, which encodes the bacteriocin mundticin; mun $B$ encoding a mundticin-translocating $\mathrm{ABC}$ transporter; and munC encoding a mundticin immunity protein. munBC expression is under independent transcriptional control from $m u n A$ and is driven by a promoter located between munA and munB and downstream of the munA terminator [34]. To enumerate gut bacteria, insects were dissected and their guts were homogenized with 5-mm steel bead using a TissueLyser (Qiagen) at $20 \mathrm{~Hz}$ for $10 \mathrm{~s}$. Homogenates were serially diluted in sterile saline and plated onto 1/10 strength TSA (Oxoid).

\section{Amplification and sequencing of 16S rRNA genes}

Bacterial colonies were categorized by morphotype and representatives were subjected to colony PCR with universal primers 27F and 1492r. Sanger sequencing of PCR products was performed by MWG Biotech (Ebersberg, Germany) or GATC (Konstanz, Germany). For highthroughput amplicon sequencing, total DNA was recovered from gut homogenates by bead milling and CTAB extraction [46] and 24 pools of DNA were constructed representing each combination of treatment and developmental stage using $100 \mathrm{ng}$ of purified DNA from each individual. Pools were subjected to PCR with barcoded versions of the universal primers $27 \mathrm{f}$ and 519r. Roche multiplex identifiers were incorporated between the sequences of adaptor $A$ and 519r to give the structure: 5'-Adaptor_A-sequencing_key-multiplex_identifier-519r-3'. PCR consisted of an initial denaturation step of $2 \mathrm{~min}$ at $94^{\circ} \mathrm{C}$ and 25 cycles of of $30 \mathrm{~s}$ at $94^{\circ} \mathrm{C}$, $20 \mathrm{~s}$ at $52^{\circ} \mathrm{C}$, and $60 \mathrm{~s}$ at $65^{\circ} \mathrm{C}$. PCR products were checked by gel electrophoresis, purified with AMPure beads, and sequenced on a 454 titanium GS FLX at 24-plex per quarter pico-titer plate. Amplicon sequence data were processed using QIIME version 1.6 [47]. Sequences were assigned to operational taxonomic units according to a $97 \%$ identity threshold using uclust [48]. Data were deposited in the NCBI SRA under accession PRJNA268795.

\section{S rRNA gene qPCR}

Based on the high-throughput 16S rRNA gene amplicon sequencing, taxon-specific 16S rRNA gene primers were used to quantify the three dominant taxa for the genera Enterococcus [49], Staphylococcus [50], and the family Enterobacteriaceae [49] in each individual. Dilution plating and high-throughput $16 \mathrm{~S}$ rRNA amplicon sequencing showed the presence of three bacterial genera: Enterococcus, Staphylococcus, and Serratia however since Serratia-specific 16S rRNA gene primers could not be designed, family-specific Enterobacteriaceae primers were used to generate individual-based qPCR measurements. Standard curves were prepared using samples derived from axenic guts spiked with known quantities of either Enterococcus mundtii G2, Staphylococcus sp, or Serratia sp. CFU. qPCR was performed on an ABI StepOne using KAPA SYBR FAST ABI Prism mastermix (Peqlab). The resulting data were log transformed and analysed using linear models in R 3.1.3.

\section{Insect rearing}

G. mellonella larvae were reared in the dark at $30^{\circ} \mathrm{C}$ on a grain-honey diet described previously [29]. Hoffman's tobacco hornworm diet was used to manipulate the G. mellonella gut microbiota. Sterile artificial diet was produced using an autoclave and heat-labile components such as Vanderzants vitamin mixture (Sigma-Aldrich) and antibiotics were dissolved in water and 
filter-sterilized before combing with molten diet at $60^{\circ} \mathrm{C}$. To remove the gut microbiota, diet was amended with $100 \mu \mathrm{g} \mathrm{ml}^{-1}$ streptomycin and tetracycline (Sigma-Aldrich). Mature finalinstar larvae were starved for $4 \mathrm{~h}$ before being transferred to sterile antibiotic-amended diet for $24 \mathrm{~h}$. Removal of the microbiota was confirmed by dissecting and plating the guts of 30 randomly-chosen larvae onto TSA plates.

To associate larvae with a specific bacterial strain, sterile diet (without antibiotics) was amended with an aliquot of an overnight culture to a final concentration of $10^{3} \mathrm{CFU} \mathrm{m}{ }^{-1}$. Larvae were removed from sterile antibiotic-amended diet, starved for $4 \mathrm{~h}$, and transferred to bacteria-amended diet for $16 \mathrm{~h}$. As is common in many Lepidoptera [32], G. mellonella adults do not possess functional mouthparts therefore oral infection of adults is not possible. Larvae were subsequently starved for $4 \mathrm{~h}$ before being returned to a conventional grain-honey diet.

\section{Plasmid segregational stability}

The segregational stability of the plasmids pRK1 and pRK62 in E. mundtii G2 was determined according to Simon and Chopin [51] in both non-selective MRS broth (Oxoid) as well as in insect hosts. To quantify stability in broth, an overnight culture was diluted in non-selective MRS broth, grown to late exponential phase and plated onto non-selective MRS agar. 384 colonies were arrayed in duplicate onto erythromycin-selective and non-selective MRS agar. To quantify stability in insects, mature larvae were mono-associated as described above with $E$. mundtii G2 carrying either pRK1 or pRK62, and returned to conventional grain-honey diet to complete larval and pupal development. The frequency of vector loss was $<2.8 \times 10^{-3}$ both in broth culture and insect hosts. In the case of broth culture, this stability is comparable to the parent vector pIL253 [51]. Upon eclosion, 10 adults carrying either mun+ or mun- strains were dissected and their guts were plated onto non-selective MRS agar (S4 Fig). 384 colonies were tested for erythromycin-sensitivity as described above. 46 randomly selected Em ${ }^{\mathrm{R}}$ colonies from each larva were screened for the presence of the plasmid by colony PCR using T7 promoter-specific primers.

\section{Determination of the stages during metamorphosis}

Complete detachment of the larval gut epithelium occurs prior to ecdysis of the larval cuticle. Therefore the staging system of Kühn and Piepho [52], as adapted by Uwo et al. [31], was used to specify the stages of midgut metamorphosis in larvae and pupae (see Uwo et al. [31] and Ellis et al. [53] for illustration). Stage I is a wandering larva that has ceased feeding and started spinning. Stemmatal pigments have not started to migrate and the midgut is empty. Stage II, is the spinning larva and pigments have started to migrate from the stemmata. Stage III, a l ater spinning larva, where the pigments have left the stemmata but are still in contact with the cuticle. The larval gut epithelium has completely detached and floats freely in the lumen. Stage IV defines a mature spinning larva and the pigments have sunk beneath the cuticle but are still visible. Stage $\mathrm{V}$ is the prepupa that has ceased spinning and stemmatal pigments are not visible. The midgut is laterally flattened and the detached larval gut has formed the yellow body which undergoes apoptosis. The new pupa is classed as stage VI; the cuticle has not sclerotized and is completely white. Stage VII describes a sclerotized pupa approximately $24 \mathrm{~h}$ after the larvalpupal molt. The midgut is cylindrical and surrounded by an extra-epithelial layer.

The migration of stemmatal pigments was monitored under a stereo microscope.

\section{RNAi}

An internal region of the cDNA sequence encoding a C-type lysozyme, previously designated lysozyme GALME (Swiss-Prot accession P82174) [54], was amplified with T7-tailed primers 
Gm_Lys_T7_F1 (5'-TAATACGACTCACTATAGGGAGAGCAAGCCGAATAAAAATGG A-3') and Gm_Lys_T7_R1 (5'-TAATACGACTCACTATAGGGAGATATCTGGCAGCGG CTTATTT-3') and used as template to synthesize dsRNA using a MEGAscript T7 Kit (Ambion). In order to knockdown lysozyme GALME expression, $500 \mathrm{ng}$ of purified dsRNA was injected into the hemocoel of mature final-instar larvae. RNAi efficacy was monitored throughout the larval-pupal molt by performing relative qPCR on cDNA derived from dissected guts using the primers Gm_Lys_qPCR_F1 (5'-ACTTTTACGAGATGCGGACTG-3') and Gm_Lys_qPCR_R1 (5'-TCTCATTCTCAACAAGGCACAC-3'), which target a region upstream of the region chosen as template for dsRNA synthesis, as well as S7e_forward and S7e_reverse which target the gene encoding ribosomal protein S7e [55], which was analyzed as a reference. Relative expression was calculated using the relative $\mathrm{Ct}$ method. cDNA was synthesized using a cDNA-Synthesis Kit H Plus (Peqlab) from $100 \mathrm{ng}$ of total RNA from a pool of RNA from 3 individual insects. qPCR was performed using a peqGOLD Hot Start-Mix kit (Peqlab) and a StepOne real-time thermocycler (Applied Biosystems) according to the manufacturer's instructions.

\section{Survival analysis}

Mature pupae were weighed and segregated individually in plastic cups covered with muslin cloth at $30^{\circ} \mathrm{C}$. Newly eclosed adults were sexed according to the forewing margin [53] and survival was recorded every $24 \mathrm{~h}$. The data were analysed in $\mathrm{R}$ with an accelerated failure time model using the survival package [56]. The Bayesian information criterion was used to select the final model.

\section{Supporting Information}

S1 Fig. C-type lysozyme gene expression during metamorphosis. Boxplots depict three biological replicates of pools of 8-10 individual insects. Wild-type (wt), grey; RNAi, white. (TIFF)

S2 Fig. Relative abundance of bacteria during host metamorphosis. Using $16 \mathrm{~S}$ rRNA gene amplicon sequencing the microbiota was sampled across metamorphosis in (A) wild-type hosts with bacteriocin-producing Entercoccus mundtii G2 symbiont, (B) immunocompromised hosts where RNAi was used to knock down lysozyme gene expression, (C) wild-type hosts with mutant Entercoccus mundtii symbionts that do not produce bacteriocin, and (D) both immunocompromised hosts and mutant symbionts. Roman numerals correspond to precise stages of the larval-pupal molt. A, adult; OTU, operational taxonomic unit. See S1 Table for full data. (TIFF)

S3 Fig. Survival of male and female Galleria mellonella. Adult individuals were derived from final-instar larvae which were cured of their microbiota using antibiotics. One group was reinoculated with E. mundtii whereas the other remained axenic. No difference in survival was detected between the two groups (Accelerated failure time model, $\mathrm{n}=106$, ANOVA, DF: 1,102, $\mathrm{p}=0.3457356)$.

S4 Fig. Densities of bacteria in Galleria mellonella adults. A, densities achieved by Enterococcus mundtii $\mathrm{G} 2$ with (mun plus) and without (mun minus) mundticin expression. Mundticin expression had no effect on CFU (negative binomial GLM, DF: 18, p = 0.4355). B, densities of different types of bacteria of newly-eclosed adults sampled from the survival experiment depicted in Fig 2.

(TIFF) 
S1 Table. OTU table.

(CSV)

S2 Table. Summary of accelerated failure time model for survival data shown in Fig 2. (PDF)

\section{Acknowledgments}

We are grateful to Jennie Garbutt, Quentin Geissmann and Jan Graßnick for technical assistance, to Christina Nielsen-LeRoux for providing G. mellonella from a long-term lab population, to Shinichi Kawamoto for providing the mun deletion mutant vectors, and to Olivia Judson for valuable comments on the manuscript.

\section{Author Contributions}

Conceived and designed the experiments: PRJ JR. Performed the experiments: PRJ. Analyzed the data: PRJ. Contributed reagents/materials/analysis tools: JR. Wrote the paper: PRJ JR.

\section{References}

1. Misof B, Liu S, Meusemann K, Peters RS, Donath a., Mayer C, et al. Phylogenomics resolves the timing and pattern of insect evolution. Science. 2014; 346: 763-767. doi: 10.1126/science.1257570 PMID: 25378627

2. Mora C, Tittensor DP, Adl S, Simpson AGB, Worm B. How many species are there on Earth and in the ocean? PLoS Biol. 2011; 9: e1001127. doi: 10.1371/journal.pbio.1001127 PMID: 21886479

3. Truman JW, Riddiford LM. The origins of insect metamorphosis. Nature. 1999; 401: 447-452. doi: 10. 1038/46737 PMID: 10519548

4. Grimaldi D, Engel M. Evolution of the Insects. Cambridge University Press; 2005.

5. Moran N. Adaptation and constraint in the complex life cycles of animals. Annu Rev Ecol Syst. 1994; 25: 573-600. Available: http://www.jstor.org/stable/2097325

6. Stansbury M, Moczek A. The evolvability of arthropods. Arthropod Biology and Evolution. Springer Berlin Heidelberg; 2013. pp. 479-493.

7. Leach JG. The method of survival of bacteria in the puparia of the seed-corn maggot (Hylernyia Cilicrura Rond.). Zeitschrift für Angew Entomol. 1934; 20: 150-161.

8. Bacot AW. On the Persistence of Bacilli in the Gut of an Insect during Metamorphosis. Trans Entomol Soc London. 1911; 497-500.

9. Bakula M. The persistence of a microbial flora during postembryogenesis of Drosophila melanogaster. J Invertebr Pathol. 1969; 14: 365-374. doi: 10.1016/0022-2011(69)90163-3 PMID: 4904970

10. Delalibera I, Vasanthakumar A, Burwitz B, Schloss PD, Klepzig KD, Handelsman J, et al. Composition of the bacterial community in the gut of the pine engraver, Ips pini (Say) (Coleoptera) colonizing red pine. Symbiosis. 2007; 43: 97-104.

11. Wong CNA, Ng P, Douglas AE. Low-diversity bacterial community in the gut of the fruitfly Drosophila melanogaster. Environ Microbiol. 2011; 13: 1889-1900. doi: 10.1111/j.1462-2920.2011.02511.x PMID: 21631690

12. Hammer TJ, McMillan WO, Fierer N. Metamorphosis of a butterfly-associated bacterial community. PLoS One. 2014; 9: e86995. doi: 10.1371/journal.pone.0086995 PMID: 24466308

13. Brucker RM, Bordenstein SR. The roles of host evolutionary relationships (genus: Nasonia) and development in structuring microbial communities. Evolution. 2012; 66: 349-362. doi: 10.1111/j.1558-5646. 2011.01454.x PMID: 22276533

14. Greenberg B. Salmonella suppression by known populations of bacteria in flies. J Bacteriol. 1969; 99 : 629-35. Available: http://www.pubmedcentral.nih.gov/articlerender.fcgi?artid=250072\&tool= pmcentrez\&rendertype=abstract PMID: 4984172

15. Greenberg B, Klowden M. Enteric Bacterial Interactions in Insects. Am J Clin Nutr. 1972; 25: 14591466. PMID: 4629542

16. Russell V, Dunn PE. Antibacterial proteins in the midgut of Manduca sexta during metamorphosis. $J$ Insect Physiol. 1996; 42: 65-71. 
17. Tebbutt $\mathrm{H}$. On the influence of metamorphosis of Musca domestica upon bacteria administered in the larval Stage. J Hyg (Lond). 1912; 12: 516-526.

18. Buchon N, Broderick NA, Lemaitre B. Gut homeostasis in a microbial world: insights from Drosophila melanogaster. Nat Rev Microbiol. 2013; 11: 615-626. doi: 10.1038/nrmicro3074 PMID: 23893105

19. Wheeler $\mathrm{D}$, Redding $\mathrm{AJ}$, Werren $\mathrm{JH}$. Characterization of an ancient lepidopteran lateral gene transfer. PLoS One. 2013; 8: e59262. doi: 10.1371/journal.pone.0059262 PMID: 23533610

20. Van Frankenhuyzen K, Liu YH, Tonon A. Interactions between Bacillus thuringiensis subsp kurstaki HD-1 and midgut bacteria in larvae of gypsy moth and spruce budworm. J Invertebr Pathol. 2010; 103 : 124-131.

21. Hernández-Martínez P, Naseri B, Navarro-Cerrillo G, Escriche B, Ferré J, Herrero S. Increase in midgut microbiota load induces an apparent immune priming and increases tolerance to Bacillus thuringiensis. Environ Microbiol. 2010; 12: 2730-2737. doi: 10.1111/j.1462-2920.2010.02241.x PMID: 20482744

22. Broderick NA, Raffa KF, Goodman RM, Handelsman J. Census of the bacterial community of the gypsy moth larval midgut by using culturing and culture-independent methods. Appl Environ Microbiol. 2004; 70: 293-300. doi: 10.1128/AEM.70.1.293 PMID: 14711655

23. Brooks MA. The Microorganisms of Healthy Insects. In: Steinhaus EA, editor. Insect Pathology: An Advanced Treatise vol 1. London: Academic Press; 1963. pp. 215-250.

24. Martin JD, Mundt JO. Enterococci in insects. Appl Microbiol. 1972; 24: 575-80. Available: http://www. pubmedcentral.nih.gov/articlerender.fcgi?artid=380616\&tool=pmcentrez\&rendertype=abstract PMID: 4628796

25. Bucher GE. Survival of populations of Streptococcus faecalis Andrewes and Horder in the gut of Galleria mellonella (Linnaeus) during metamorphosis, and transmission of the bacteria to the filial generation of the host. J Insect Pathol. 1963; 5: 336-343.

26. Xiang H, Wei G, Jia S, Huang J, Miao X, Zhou Z, et al. Microbial communities in the larval midgut of laboratory and field populations of cotton bollworm (Helicoverpa armigera). Can J Microbiol. 2006; 52: 1085-1092. doi: 10.1139/W06-064 PMID: 17215900

27. Raymond B, Johnston PR, Wright DJ, Ellis RJ, Crickmore N, Bonsall MB. A mid-gut microbiota is not required for the pathogenicity of Bacillus thuringiensis to diamondback moth larvae. Environ Microbiol. 2009; 11: 2556-2563. doi: 10.1111/j.1462-2920.2009.01980.x PMID: 19555371

28. Jarosz J. Gut flora of Galleria mellonella suppressing ingested bacteria. J Invertebr Pathol. 1979; 34: 192-198. PMID: 119813

29. Johnston PR, Crickmore N. Gut bacteria are not required for the insecticidal activity of Bacillus thuringiensis toward the tobacco hornworm, Manduca sexta. Appl Environ Microbiol. 2009; 75: 5094-5099. doi: 10.1128/AEM.00966-09 PMID: 19525273

30. Raymond B, Johnston PR, Nielsen-LeRoux C, Lereclus D, Crickmore N. Bacillus thuringiensis: an impotent pathogen? Trends Microbiol. 2010; 18: 189-94. doi: 10.1016/j.tim.2010.02.006 PMID: 20338765

31. Uwo MF, Ui-Tei K, Park P, Takeda M. Replacement of midgut epithelium in the greater wax moth, Galleria mellonela, during larval-pupal moult. Cell Tissue Res. 2002; 308: 319-31. doi: 10.1007/s00441002-0515-1 PMID: 12037588

32. Tammaru T, Haukioja E. Capital breeders and income breeders among Lepidoptera: consequences to population dynamics. Oikos. 1996; 77: 561-564. doi: $10.2307 / 3545946$

33. Zdybicka-Barabas A, St czek S, Mak P, Skrzypiec K, Mendyk E, Cytryńska M. Synergistic action of Galleria mellonella apolipophorin III and lysozyme against Gram-negative bacteria. Biochim Biophys Acta. 2013; 1828: 1449-56. doi: 10.1016/j.bbamem.2013.02.004 PMID: 23419829

34. Kawamoto S, Shima J, Sato R, Eguchi T, Ohmomo S, Shibato J, et al. Biochemical and Genetic Characterization of Mundticin KS, an Antilisterial Peptide Produced by Enterococcus mundtii NFRI 7393. Appl Env Microbiol. 2002; 68: 3830-3840. doi: 10.1128/AEM.68.8.3830

35. Ebert D. The Epidemiology and Evolution of Symbionts with Mixed-Mode Transmission. Annu Rev Ecol Evol Syst. 2013; 44: 623-643. doi: 10.1146/annurev-ecolsys-032513-100555

36. Koch H, Schmid-Hempel P. Socially transmitted gut microbiota protect bumble bees against an intestinal parasite. Proc Natl Acad Sci U S A. 2011; 108: 19288-92. doi: 10.1073/pnas.1110474108 PMID: 22084077

37. Vigneron A, Masson F, Vallier A, Balmand S, Rey M, Vincent-Monégat C, et al. Insects recycle Endosymbionts when the benefit is over. Curr Biol. 2014; 24: 2267-2273. doi: 10.1016/j.cub.2014.07.065 PMID: 25242028

38. Stoll S, Feldhaar H, Fraunholz MJ, Gross R. Bacteriocyte dynamics during development of a holometabolous insect, the carpenter ant Camponotus floridanus. BMC Microbiol. 2010; 10: 308. doi: 10.1186/ 1471-2180-10-308 PMID: 21122115 
39. Nicholson D, Ross A, Mayhew P. Fossil evidence for key innovations in the evolution of insect diversity. Proc R Soc B Biol Sci. 2014; 281: 1783. Available: http://rspb.royalsocietypublishing.org/content/281/ 1793/20141823.short

40. Arendt J. Adaptive intrinsic growth rates: an integration across taxa. Q Rev Biol. 1997; 72: 149-177.

41. Godfray H. Parasitoids: behavioral and evolutionary ecology. Princeton University Press; 1994.

42. Lazzaro BP, Rolff J. Danger, microbes, and homeostasis. Science. 2011; 332: 43-44. doi: 10.1126/ science.1200486 PMID: 21454776

43. Fedhila S, Buisson C, Dussurget $O$, Serror $P$, Glomski IJ, Liehl P, et al. Comparative analysis of the virulence of invertebrate and mammalian pathogenic bacteria in the oral insect infection model Galleria mellonella. J Invertebr Pathol. 2010; 103: 24-29. doi: 10.1016/j.jip.2009.09.005 PMID: 19800349

44. Dunny GM, Lee LN, LeBlanc DJ. Improved electroporation and cloning vector system for gram-positive bacteria. Appl Environ Microbiol. 1991; 57: 1194-201. Available: http://www.pubmedcentral.nih.gov/ articlerender.fcgi? artid=182867\&tool=pmcentrez\&rendertype=abstract PMID: 1905518

45. Hébert L, Courtin P, Torelli R, Sanguinetti M, Chapot-Chartier MP, Auffray Y, et al. Enterococcus faecalis constitutes an unusual bacterial model in lysozyme resistance. Infect Immun. 2007; 75: 5390-5398. doi: 10.1128/IAI.00571-07 PMID: 17785473

46. Ausubel FM, Brent R, Kingston RE, Moore DD, Seidman JG, Smith J a, et al. Current Protocols in Molecular Biology. Molecular Biology. 2003.

47. Caporaso JG, Kuczynski J, Stombaugh J, Bittinger K, Bushman FD, Costello EK, et al. QIIME allows analysis of high-throughput community sequencing data. Nature methods. 2010; 7: 335-336. doi: 10 . 1038/nmeth.f.303 PMID: 20383131

48. Edgar RC. Search and clustering orders of magnitude faster than BLAST. Bioinformatics. 2010; 26: 2460-2461. doi: 10.1093/bioinformatics/btq461 PMID: 20709691

49. Hermann-Bank ML, Skovgaard K, Stockmarr A, Larsen N, Mølbak L. The gut microbiotassay: a highthroughput qPCR approach combinable with next generation sequencing to study gut microbial diversity. BMC Genomics. 2013; 14: 788. doi: 10.1186/1471-2164-14-788 PMID: 24225361

50. Arboleya S, Binetti A, Salazar N, Fernández N, Solís G, Hernández-Barranco A, et al. Establishment and development of intestinal microbiota in preterm neonates. FEMS Microbiol Ecol. 2012; 79: 763772. doi: 10.1111/j.1574-6941.2011.01261.x PMID: 22126419

51. Simon D, Chopin A. Construction of a vector plasmid family and its use for molecular cloning in Streptococcus lactis. Biochimie. 1988; 0: 559-66. Available: http://www.ncbi.nlm.nih.gov/pubmed/2844302 PMID: 2844302

52. Kühn A, Piepho H. Über hormonale Wirkungen bei der Verpuppung der Schmetterlinge. Nachrichten von der Gesellschaft der Wissenschaften zu Göttingen. 1936; 2: 141-154.

53. Ellis JD, Graham JR, Mortensen A. Standard methods for wax moth research. J Api Res. 2013; 52: 118. doi: 10.3896/IBRA.1.52.1.10

54. Vogel $\mathrm{H}$, Altincicek B, Glöckner $\mathrm{G}$, Vilcinskas A. A comprehensive transcriptome and immune-gene repertoire of the lepidopteran model host Galleria mellonella. BMC Genomics. 2011; 12: 308. doi: 10. 1186/1471-2164-12-308 PMID: 21663692

55. Wojda I, Jakubowicz T. Humoral immune response upon mild heat-shock conditions in Galleria mellonella larvae. J Insect Physiol. 2007; 53: 1134-1144. doi: 10.1016/j.jinsphys.2007.06.003 PMID: 17631308

56. Therneau T. A Package for Survival Analysis in S. R package version 2.37-7 [Internet]. Survival. 2014. Available: http://cran.r-project.org/package=survival 